\title{
Modulation of spatial attention by fear-conditioned stimuli: an event-related fMRI study
}

\author{
Jorge L. Armony ${ }^{\mathrm{a}, \mathrm{b}, *}$, Raymond J. Dolan ${ }^{\mathrm{a}, \mathrm{c}}$ \\ ${ }^{a}$ Wellcome Department of Cognitive Neurology, Institute of Neurology, 12 Queen Square, London WC1N 3BG, UK \\ ${ }^{\mathrm{b}}$ Institute of Cognitive Neuroscience, University College London (UCL), Alexandra House, 17 Queen Square, London WC1N 3AR, UK \\ ${ }^{\mathrm{c}}$ The Royal Free Hospital School of Medicine, Rowland Street, London NW3 2PF, UK
}

Received 6 March 2001; accepted 24 August 2001

\begin{abstract}
Stimuli that signal threat can capture subjects' attention, leading to more efficient detection of, and faster responses to, events occurring in that part of the environment. In the present study we explored the behavioural and anatomical correlates of the modulation of spatial attention by emotion using a fear conditioning paradigm, combined with a covert spatial orienting task. Reaction times for the detection of a peripheral target, which was preceded by brief $(50 \mathrm{~ms})$ presentations of the visual conditioned stimulus $(\mathrm{CS}+)$ in either the same or opposite visual field, showed an interaction between stimulus emotionality and attention shifts. We used event-related functional magnetic resonance imaging (fMRI) to characterise the associated neural responses. Consistent with previous studies, conditioning-induced enhanced responses were observed in the amygdala and extrastriate visual cortex. The modulation of spatial attention by a conditioned stimulus was associated with enhanced activity in regions of frontal and parietal cortices previously implicated in spatial attention, as well as in the lateral orbitofrontal cortex (IOFC). (C) 2002 Elsevier Science Ltd. All rights reserved.
\end{abstract}

Keywords: Amygdala; Fear conditioning; Neuroimaging; Orbitofrontal cortex; Neurophysiology

\section{Introduction}

Detection of danger and rapid elicitation of appropriate defence reactions are crucial for survival. The fear system, highly conserved throughout evolution, operates in a rapid and efficient fashion, in some cases even without conscious awareness of an eliciting stimulus [21]. In many instances, however, once danger is detected and the initial automatic fear responses elicited, further action is necessary, requiring the redirection of attentional resources, with the engagement of flexible response repertoires, towards the threatening stimulus. This influence of emotionality on spatial attention in humans has been investigated using a variety of behavioural tasks (e.g. [3,28,38,39,54]). However, although much progress has been made in characterising neural circuits underlying fear processing (for reviews see $[4,23,34]$ ) and spatial attention (e.g. $[13,14,27,37,52]$ ), little is known about how these two systems interact in humans.

\footnotetext{
* Corresponding author. Tel.: +44-20-7679-5431; fax: +44-20-7916-8517.

E-mail address: j.armony@ucl.ac.uk (J.L. Armony).
}

Attention and emotion can interact either by attention influencing emotional processing, or emotion modulating attentional processing. In the present study we used event-related functional magnetic resonance imaging (fMRI) to address the question of how emotion, specifically fear, influences attention, by employing a paradigm that combined discriminatory fear conditioning with a covert spatial orienting task. During scanning, subjects viewed pictures of two angry faces, one of which was paired (conditioned stimulus $(\mathrm{CS}+)$ ), and the other not paired (CS-), with a loud burst of white noise, the unconditioned stimulus (US). We hypothesised that the $\mathrm{CS}+$, having acquired aversive emotional value through conditioning, would automatically capture subjects' attention. We tested this hypothesis with a modified version of the well-known dot-probe covert attention task [47]. Subjects were instructed to detect the location of a target, appearing on either side of the central fixation location. In the critical experimental condition, the dot target was preceded by a brief presentation of a CS + and CS - side by side, but in opposite visual fields. If attention was captured by the $\mathrm{CS}+$, detection of targets on the opposite side (incongruent trials) should be slower than those on the same side (congruent trials). That is, a difference in reaction times between congruent 


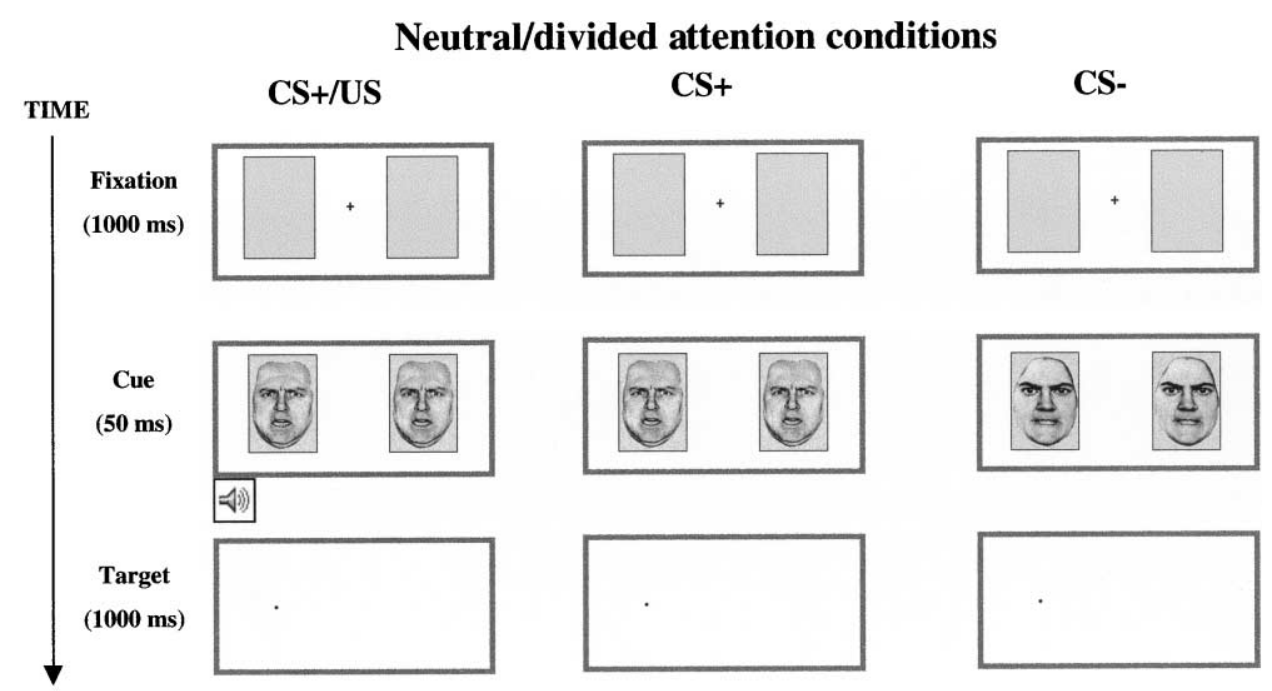

\section{Focused attention conditions}

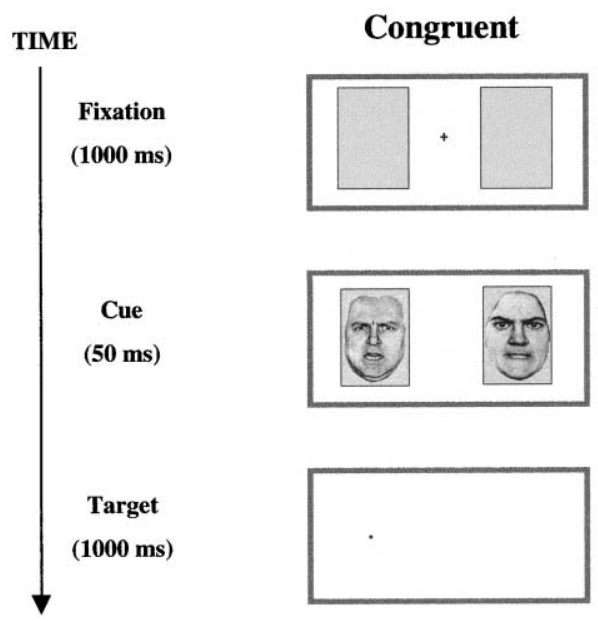

\section{Incongruent}
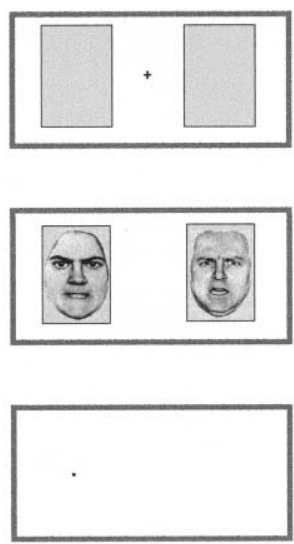

Fig. 1. Schematic illustration of the five trial types used in the study (see Section 2 for details). The fixation cross was present for $1 \mathrm{~s}$, and the face stimuli (cue) appeared for $50 \mathrm{~ms}$, immediately followed by the target. In the neutral/divided attention conditions, the same stimulus (either CS + or $\mathrm{CS}-$ ) was presented on both sides, whereas in the focused-attention conditions the CS + and CS - were presented together, side by side; in congruent trials the location of target was the same as that of the CS+, and opposite in the incongruent trials. In the CS+/US condition, a loud burst of white noise $(200 \mathrm{~ms})$ was presented in conjunction with the face stimuli.

and incongruent trials would suggest that indeed spatial attention was modulated by the affective value of the stimuli. Furthermore, by comparing neural responses between these trials where, according to our hypothesis, attention would be captured by the $\mathrm{CS}+$ (henceforth, referred as focused-attention conditions) with trials in which the same face stimulus, either the $\mathrm{CS}+$ or the $\mathrm{CS}-$, was presented on both sides-therefore, attention being equally allocated to both hemifields (the neutral/divided attention conditions; see Fig. 1), we were able to determine which brain regions were involved in this modulation of attention by emotion.

\section{Materials and methods}

\subsection{Subjects}

Ten healthy volunteers, without a history of neurological or psychiatric impairments, participated in this study. All subjects provided written informed consent before the experiment. Data from four subjects were excluded from the analysis due to technical problems with the scanner and/or the stimulus delivery system. The results presented here correspond to data obtained form the remaining six subjects (three male, three female). All procedures were approved by 
the Joint Ethics Committee of National Hospital and Institute of Neurology.

\subsection{Experimental paradigm}

Fig. 1 shows a schematic of the different trial types. All trials began with a fixation cross in the middle of the screen for $1 \mathrm{~s}$, followed by the presentation of two faces, shown for $50 \mathrm{~ms}$. Immediately after the offset of the face stimuli, a small dot was presented on either side of the visual field, coinciding with the location of one of the two previously shown faces. Subjects were required to respond, as quickly and accurately as possible, to the location of the probe, using a keypad with their right hand. Subjects were instructed to ignore the face stimuli and concentrate on the target detection task.

Two angry faces, one male and one female, taken from the Ekman and Friesen series [20] were used as CS+ and CS-. The use of angry faces as CS has been shown to provide strong conditioning responses, both at the neural [44] and autonomic [21] level, and be less susceptible to extinction than other stimuli. Because both the $\mathrm{CS}+$ and $\mathrm{CS}-$ had similar affective value at the onset of the experiment, differential responses $(\mathrm{CS}+>\mathrm{CS}-)$ can be attributed to the effects of conditioning over and above any responses elicited by the intrinsic nature of the stimuli. Furthermore, the assignment of the two stimuli as either $\mathrm{CS}+$ or $\mathrm{CS}-$ was counterbalanced across subjects. We used a 50\% partial reinforcement schedule (i.e. only half of the presentations of the $\mathrm{CS}+$ were paired with the US) to allow us to investigate the haemodynamic response to the $\mathrm{CS}+$ in the absence of the US (see $[5,6])$. The US, a $200 \mathrm{~ms}$ burst of white noise, was delivered through plastic tubes, sealed by foam ear inserts and further shielded by plastic ear defenders, to minimise the influence of the gradient switching noise from the scanner. The amplitude of the US was set individually by each subject, following the instructions that it should be aversive but not painful. The final amplitude used was very similar for all the subjects $(\sim 100 \mathrm{~dB})$.

The face stimuli consisted of two faces presented side by side. There were five types of trials used during the experiment: $\mathrm{CS}+/ \mathrm{US}, \mathrm{CS}+, \mathrm{CS}-$, incongruent, and congruent (Fig. 1). The $\mathrm{CS}+/ \mathrm{US}, \mathrm{CS}+$ and $\mathrm{CS}-$ presentations consisted of the same face presented on both sides. In the other two critical trial types, the $\mathrm{CS}+$ and $\mathrm{CS}-$ were presented side by side; congruent trials refer to the cases when the location of the target coincided with the side where the CS+ had been presented while incongruent trials refer to instances where the relative position of the target and the CS+ was in opposition. During these trials, the US was never presented, in order to avoid the possibility of conditioning to the CS- (by itself or through conditioning to the CS+/CScompound stimulus).

In total, there were 192 trials: $46 \mathrm{CS}+(30$ paired and 16 alone), $46 \mathrm{CS}-, 50$ congruent, and 50 incongruent trials. The average trial onset asynchrony was $7 \pm 2.5 \mathrm{~s}$. The location of the target with respect to visual hemifield was randomised, and equally distributed within each trial type. Subjects recorded their responses using two buttons (left and right) in a keypad. For the analysis of reaction times (RTs), error trials were discarded $(<3 \%)$. Median values of RTs in each condition were computed for each subject.

\subsection{Image acquisition and data analysis}

Images were acquired with a $2 \mathrm{~T}$ Magnetom VISION whole-body MRI system (Siemens, Erlangen, Germany) equipped with a head volume coil. T2*-weighted echoplanar image volumes with blood oxygenation level-dependent (BOLD) contrast (echo time, $40 \mathrm{~ms} ; 64 \times 64$ pixels) were acquired in an axial orientation. Each volume comprised of 32 slices (slice thickness, $3 \mathrm{~mm}$ ), positioned to cover the whole brain. The effective repetition time $\left(T_{R}\right)$ was $3.2 \mathrm{~s} /$ vol. To minimise head motion, subjects were restrained with bitemporal pressure pads. A total of 460 volumes were acquired for each subject, over $30 \mathrm{~min}$.

Image processing and statistical analysis were performed using SPM99 (Wellcome Department of Cognitive Neurol$\operatorname{ogy}{ }^{1}$ ) $[26,61]$. The imaging time series was realigned to the first volume to correct for interscan movement. To account for the difference in sampling time of different slices, voxel time series were interpolated using sinc interpolation and resampled using the slice at the anterior-posterior commissural line as reference. Finally, the functional images were spatially normalised to a standard Talairach space [57] based on a template provided by the Montreal Neurological Institute [22] to allow group analysis. A T1-weighted anatomical MRI $(1 \mathrm{~mm} \times 1 \mathrm{~mm} \times 1.5 \mathrm{~mm}$ voxel slice $)$ was obtained for each subject and coregistered with the mean realigned functional image and normalised using the parameters determined for the functional images. A mean anatomical image was created from the subjects' individual scans, onto which activations were overlaid for anatomical localisation. Functional data were smoothed using a $8 \mathrm{~mm}$ (full-width at half maximum (FWHM)) isotropic Gaussian kernel to compensate for residual inter-subject variability and to allow for the application of Gaussian random field theory in the statistical analysis [26].

Data were analysed by modelling the evoked haemodynamic responses for the different stimuli as delta functions convolved with a synthetic haemodynamic function (hrf) and its temporal derivative (hrft), in the context of the fixed-effects general lineal model $[25,30]$. We defined five event types: $\mathrm{CS}+/ \mathrm{US}, \mathrm{CS}+$ (alone), $\mathrm{CS}-$, incongruent, and congruent (Fig. 1). Differential effects were tested by applying appropriate linear contrasts to the parameter estimates for the hrf and hrft regressors of each event, resulting in a $t$-statistic for each voxel. These $t$-statistics (transformed to $Z$-statistics) constitute a statistical parametric map (SPM).

\footnotetext{
${ }^{1}$ http://www.fil.ion.ucl.ac.uk.
} 
Three contrasts were calculated. The first one, $\mathrm{CS}+$ versus $\mathrm{CS}-$, was aimed at detecting those areas involved in the conditioning paradigm. The second contrast involved comparing the focused-attention trials (i.e. congruent and incongruent) with the neutral/divided attention trials $(\mathrm{CS}+$ and $\mathrm{CS}-$ ). In this way, we aimed to identify areas correlated with the modulation of focused spatial attention by the $\mathrm{CS}+$, while removing the influence of the presentation of the $\mathrm{CS}+$ per se (hence, the inclusion of the $\mathrm{CS}+$ trials in the comparison). The third contrast consisted in the comparison between incongruent and congruent trials. The corresponding $P$-values were corrected for multiple comparisons across the entire brain, except where otherwise indicated.

In the case of the amygdala, we applied a small volume correction [61] based on our a priori hypothesis of its involvement in fear conditioning. The volume of interest con- sisted in a $8 \mathrm{~mm}$ sphere centred on the amygdala coordinates reported in a previous fMRI study of fear conditioning [5]. The size and shape of this volume was the same as in previous studies from our laboratory $[5,6,42,58]$ and based on the size of the amygdala $[12,24,48]$ and the spatial smoothing of the functional images ( $8 \mathrm{~mm}$ FWHM Gaussian kernel).

\section{Results}

\subsection{Fear conditioning}

In order to assess the effects of fear conditioning on neural activity, we compared the responses evoked by the $\mathrm{CS}+$ to those evoked by the CS- across the session. In this comparison, we excluded those trials in which the US was
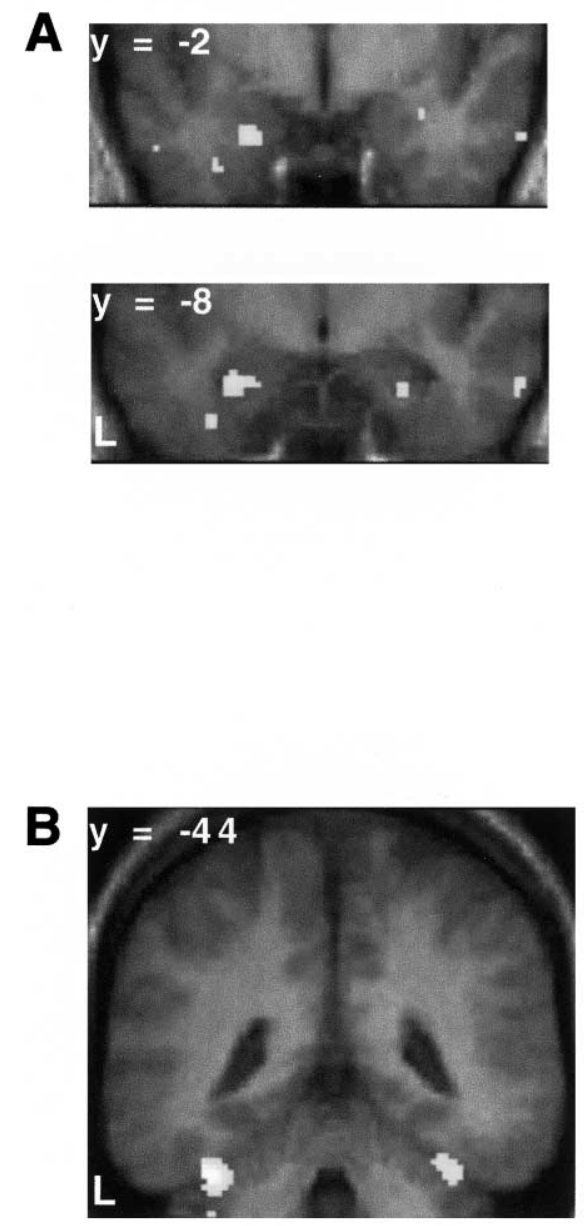
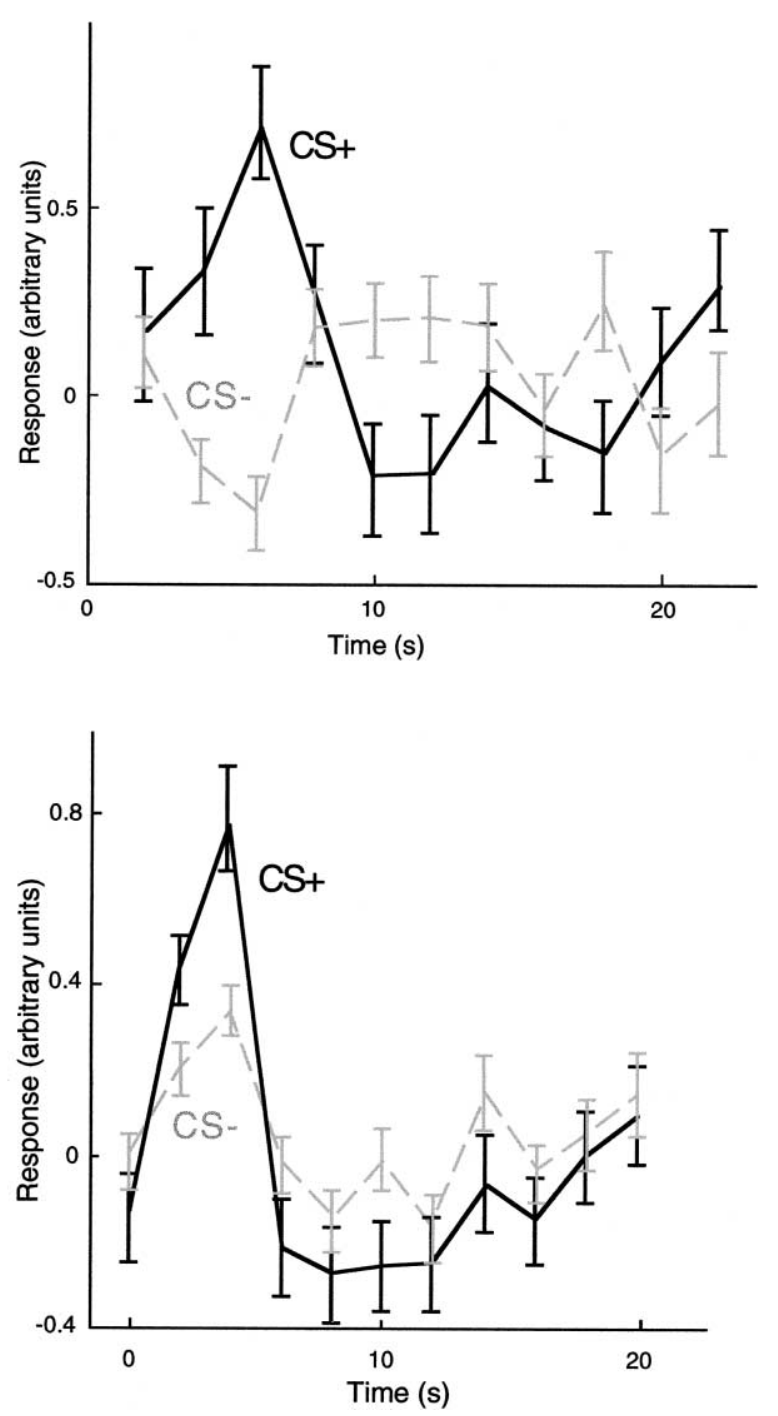

Fig. 2. Left: coronal slices depicting the activation of anterior and posterior amygdala (A) and fusiform gyrus (B) in CS+ compared to CS- trials derived from the group analysis, overlaid onto the mean anatomical MR image of all subjects, normalised to Talairach space using the MNI template. The coordinates and significance of the activations are given in Table 1. Right: group-averaged peri-stimulus time responses of the left amygdala voxel (A) and left fusiform gyrus (B) in response to the $\mathrm{CS}+$ (black) and $\mathrm{CS}-$ (grey). 
Table 1

Areas showing differential activations for the conditioned stimulus (CS+), compared to the CS-

\begin{tabular}{lll}
\hline Brain area & Coordinates $(\mathrm{mm})$ & $Z$-score \\
\hline Right inferior occipital gyrus & $36,-78,-14$ & 4.69 \\
Left inferior occipital gyrus & $-36,-62,-22$ & 4.35 \\
Right fusiform gyrus & $40,-62,-24$ & 4.19 \\
Left fusiform gyrus & $-38,-44,-28$ & $4.87^{*}$ \\
Right dorsal pons & $4,-32,-42$ & $5.10^{*}$ \\
Right anterior amygdala & $30,0,-20$ & $3.46^{* *}$ \\
Right posterior amygdala & $26,-8,-26$ & $3.45^{* *}$ \\
Left anterior amygdala & $-24,-2,-28$ & $4.09^{* *}$ \\
\hline
\end{tabular}

${ }^{*} P$-value corrected for multiple comparisons within the entire brain volume.

** $P$-value corrected for multiple comparisons within a volume of interest based on [5].

delivered (i.e. the CS+/US trials, see Fig. 1), thus, removing the potential confound of US-elicited neural responses from the analysis (see [6]). This analysis revealed a statistically significant bilateral differential activations within the anterior amygdala and the right posterior amygdala, as well as bilateral activation in extrastriate regions centred on the inferior occipital gyrus and posterior fusiform cortex, and in the right dorsal pons (pontine tegmentum). The anatomical coordinates and significance levels of these activations are shown in Table 1, and the location of the amygdala activations is shown in Fig. 2A (left), overlaid on the subjects' mean normalised anatomical image. Fig. 2A (right) shows the peri-stimulus time courses for the $\mathrm{CS}+$ and $\mathrm{CS}-$ for the left amygdala voxel. Fig. 2B (left) shows the anatomical locations of the activations in fusiform gyrus, whereas Fig. 2B (right) depicts the corresponding evoked responses to the
$\mathrm{CS}+$ and $\mathrm{CS}-$ for the maximum voxel in the left fusiform gyrus.

\subsection{Orienting of attention}

\subsubsection{Behaviour}

Reaction times for target detection as a function of the event type are shown in Fig. 3. Subjects were significantly slower to respond to targets during incongruent trials, that is, when the CS+ and probe appeared on opposite sides of the visual display than during congruent trials (Wilcoxon test, $Z=1.99, P<0.05)$. There was no significant difference between reaction times to the $\mathrm{CS}+$ and to the $\mathrm{CS}-$ alone $(Z<1)$.

These results show that, as predicted, subjects' spatial attention was preferentially captured by the CS+. Given that the two stimuli used (angry faces) were equivalent in their salience and intrinsic affective value, and their assignment as CS+ or CS- counterbalanced across subjects, we can conclude that the capture of attention by the CS+ was due to its acquired aversive affective value through Pavlovian fear conditioning

\subsubsection{Neuroimaging}

Because we were particularly interested in the neural correlates of the modulation of spatial attention by the $\mathrm{CS}+$, we collapsed, in the first instance, the congruent and incongruent trials, as in both cases subjects' attention was captured by the CS+ regardless of the location of the target. That is, we compared the two focused-attention conditions (congruent and incongruent) with the neutral/divided attention conditions (CS+ and CS-; see Fig. 1). We also analysed the neural activations associated with the comparison between congruent and incongruent trials, as described

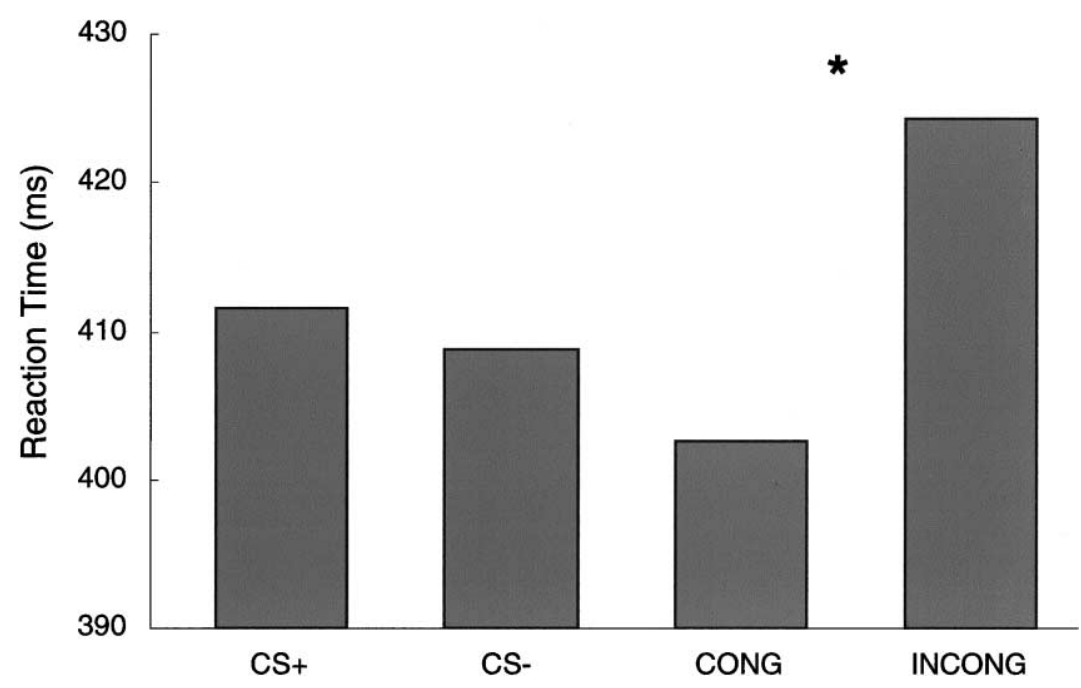

Fig. 3. Group-averaged reaction times to the peripheral target for the CS+, CS - , congruent (CS+ left, target left; CS + right, target right) and incongruent $(\mathrm{CS}+$ left, target right; CS+ right, target left) trials. Subjects were significantly slower in responding to the target in incongruent trials than in congruent ones $\left({ }^{*} P<0.05\right)$. 
Table 2

Areas of significant differential activation during the focused-attention conditions (congruent and incongruent), compared to the neutral/divided attention conditions $(\mathrm{CS}+, \mathrm{CS}-)^{\mathrm{a}}$

\begin{tabular}{lll}
\hline Brain area & Coordinates $(\mathrm{mm})$ & $Z$-score \\
\hline Left SMA/anterior cingulate & $-12,-2,60$ & $5.58^{*}$ \\
Right SMA/anterior cingulate & $4,12,58$ & $6.18^{*}$ \\
Left frontal eye fields & $-28,-16,62$ & $5.20^{*}$ \\
Right frontal eye fields & $40,-2,62$ & 4.67 \\
Left anterior IPS/precentral sulcus & $-40,-34,46$ & $4.86^{*}$ \\
Right anterior IPS/precentral sulcus & $40,-32,36$ & 4.05 \\
Left IPS & $-32,-50,58$ & $5.46^{*}$ \\
Right IPS & $42,-48,54$ & 3.82 \\
Left orbitofrontal cortex & $-32,46,-6$ & $4.86^{*}$ \\
Right orbitofrontal cortex & $30,50,-6$ & 4.15 \\
\hline
\end{tabular}

a SMA: supplementary motor area; IPS: intraparietal sulcus.

* $P$-value corrected for multiple comparisons within the entire brain volume.

below. Table 2 shows the anatomical locations and levels of significance in areas more active in the conditions involving focused spatial attention. The locations of these activations are shown in a rendered canonical brain in Fig. 4A. We observed differential activation of bilateral supplementary motor area (SMA)/anterior cingulate, left parietal cortex (IPS, extending into the post-central sulcus) and left frontal eye fields (extending into the precentral sulcus). There was also activation of the corresponding areas on the right hemisphere, although they did not reach the significance criterion of $P<0.05$ corrected (all areas $P<0.001$ uncorrected; see Table 2).

In addition, we observed significant activation of lateral orbitofrontal cortex (1OFC) in the left hemisphere and, to a lesser degree, in the right (Fig. 4B and Table 2). This activation, centred on the anterior aspects of BA11, corresponding to the anterior orbital gyrus $[10,11]$, was specific to the focused-attention trials, and was equally expressed in the congruent and incongruent trials, as confirmed by an inspection of the corresponding parameter estimates obtained from the analysis. Furthermore, in a contrast of each of the focused-attention conditions separately (i.e. congruent and incongruent) with the neutral/divided attention trials, we obtained similar magnitudes of activations in 1OFC in both cases (congruent: $Z=4.5$, incongruent: $Z=3.9$ ). A contrast of the simple main effects of the neutral/divided conditions did not reveal activation in this area, even when we adopted a less stringent significance threshold $(P<0.01$ uncorrected).

Previous studies $[14,27,46]$ have reported an interaction between laterality of activations and direction of attention shift, particularly in parietal cortex. Specifically, activations in a given hemisphere are more pronounced when attention is directed to the contralateral visual field, especially for the left hemisphere. We explicitly tested this prediction by dividing the focused-attention trials based
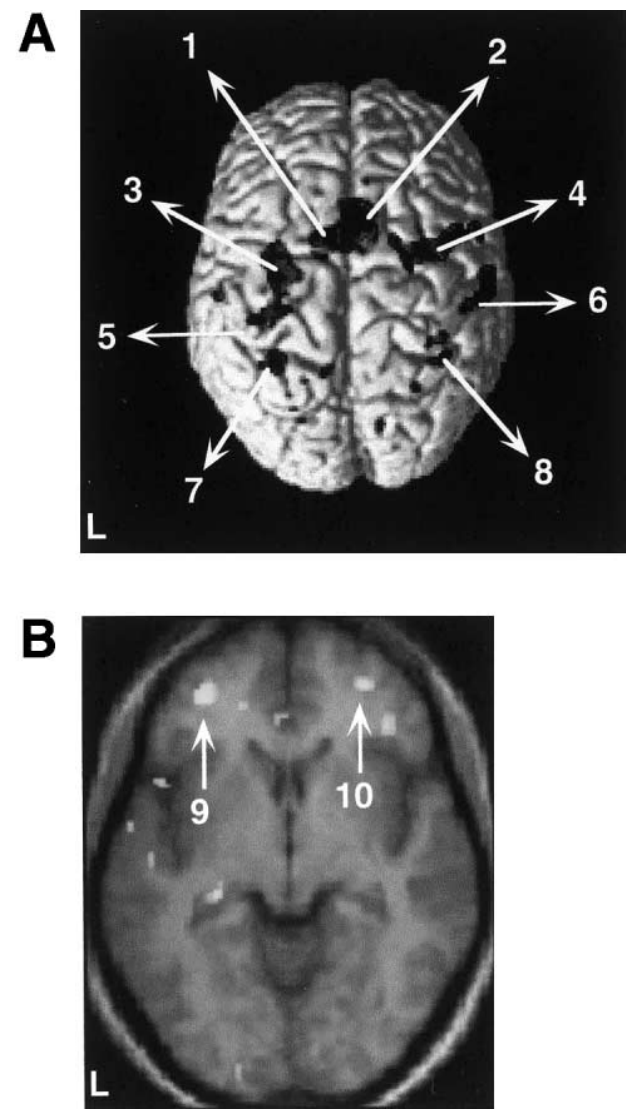

Fig. 4. (A) Dorsal view of a T1-weighted anatomical template normalised into Talairach space, showing the cortical regions displaying increased activation in the focused-attention trials, compared to the neutral/divided attention ones (see Fig. 1). (B) Differential activation of the orbitofrontal cortex in the focused-attention trials compared to the neutral/divided attention conditions. The activations are overlaid Talairach onto the average anatomical MR image of all subjects, normalised to Talairach space using the MNI template. Images are thresholded at $P<0.001$ uncorrected for visualisation. Numbers correspond to the areas reported in Table 2.

on the laterality of the $\mathrm{CS}+$, independently of target position.

Trials in which the CS+ was presented on the left visual field, compared to neutral/divided attention trials, resulted in significant parietal activations in the right hemisphere $(x=$ $-44, y=-38, z=48, Z=3.75, P<0.001$ uncorrected), whereas right $\mathrm{CS}+$ presentations significantly activated left and right parietal cortices (left: $x=36, y=-38, z=38$, $Z=3.91, P<0.001$ uncorrected; right: $x=38, y=-30$, $z=40, Z=3.50, P<0.001$ uncorrected).

Finally, we investigated the differences in haemodynamic responses between congruent and incongruent trials. A contrast between incongruent and congruent conditions revealed no differentially activated regions after correcting for multiple comparisons across the brain. However, given that in an fMRI study of spatial attention Nobre and co-workers $[15,45]$ reported orbitofrontal activations associated exclusively with incongruent trials, we specifically explored the possibility of similar activations in our study. Consistent with 
their findings, we observed activation in left lateral OFC $(x=-24, y=30, z=-18 ; Z=3.32, P<0.001)$, close to the location $(x=-30, y=30, z=-18)$ reported by Coull et al. [15]. It is important to point out that the anatomical location of this activation, located in the posterior orbital gyrus [10], is distinct from the ones associated with the focused-attention trials (Table 2 and Fig. 4B), which were present in both congruent and incongruent trials (see earlier sections).

\section{Discussion}

\subsection{Conditioning-induced differential responses}

The differential amygdala activation, extending into neighbouring perirhinal and entorhinal cortices, in response to the CS+ observed in this study is in agreement with previous human functional neuroimaging studies [5,6,33,44] and single-unit recordings in rodents (e.g. [49,50]) during fear conditioning.

Unlike some previous fMRI conditioning studies [5,6,33], the activations elicited by the $\mathrm{CS}+$ did not decrease over the course of the experiment. This finding is consistent with a recent study by Morris et al. [42], showing a possible dissociation in the temporal patterns of CS-related activations within the amygdala. Specifically, Morris et al. found that responses in the ventral amygdala, in a location close to the one found in our study, did not show time-related decreases, whereas such time changes were observed in the dorsal amygdala. Although the reasons for the differences between studies are yet not clear, Morris et al. suggested that the discrepancy could be related to the nature of the conditioned stimuli. In their study, they used angry faces, which have been shown to facilitate the acquisition and maintenance of conditioned responses [21]. In contrast, the previous studies used either neutral faces [6] or colour patches [33]. The present findings are consistent with this hypothesis, as we also used angry faces as conditioned stimuli. Other explanations, based on task-related differences, are also possible. Whereas in previous studies, subjects were passively exposed to the CSs, in our study they were asked to perform a reaction time task, which demanded sustained attention, and could, thus, have altered the level of general arousal. It is important to point out, however, that the coexistence of time-dependent and time-independent amygdala conditioning-induced increased responses to the $\mathrm{CS}+$ is in agreement with single-unit studies in rodents, showing distinct population of lateral amygdala cells with differential time courses of responses [49-51].

The differential activation associated with $\mathrm{CS}+$ trials observed in the dorsal pons, in what appears to be the caudal pontine reticular nucleus $(\mathrm{PnC})$, is consistent with its role in the acoustic startle reflex (for reviews see [18-31]). In the present study, the visual $\mathrm{CS}+$ was associated with the expectation of a loud burst of white noise, the US, whereas the
CS - was not. Previous studies have shown that the startle reflex can be potentiated by the presence of a visual conditioned stimulus, by way of direct projections of the central nucleus of the amygdala to the PnC (see [19]). Thus, the activation observed here may be associated with the preparation of motor responses in response to an aversive stimulus. Although no auditory US was presented in the trials analysed in this contrast, expectation of its presentation could lead to an enhanced response of the reticular formation, by way of direct projections from the central nucleus of the amygdala [53].

Finally, the differential activation in sensory cortical areas (e.g. fusiform gyrus) in response to the $\mathrm{CS}+$ is consistent with previous neuroimaging $[6,43]$ and electrophysiological $[2,49,59]$ studies, supporting the notion that fear conditioning can modulate the cortical processing of sensory information, probably by way of direct and indirect feedback projections from the amygdala $[1,2,35]$.

\subsection{Modulation of spatial attention by conditioned fear stimuli}

The main objective of the present study was to assess, both behaviourally and neurophysiologically, whether stimuli that acquire aversive value, through classical conditioning, modulate spatial attention. The behavioural results, shown in Fig. 3, demonstrate that conditioned stimuli can indeed capture subjects' attention. Our findings are entirely consistent with previous behavioural studies showing similar effects employing naturally threatening stimuli, such as angry faces (compared to neutral ones) [38]. This study, however, has the advantage that the stimuli used had an intrinsically equivalent affective value prior to the experiment; the only difference was that one of them was consistently associated with an unconditioned aversive stimulus. Because of this, we can rule out the possibility that the attention effects were due to physical differences between the stimuli, rather than their emotional value.

The lack of a significant difference in reaction times between the $\mathrm{CS}+$ and $\mathrm{CS}$ - trials is perhaps somewhat surprising. It could be argued that the presentation of the CS+ in both hemifields should have resulted in an enhancement in divided attention and, thus, result in faster response times to the target than in the case of $\mathrm{CS}$ - presentations. One possible explanation for the negative finding is that because all trials were preceded by a fixation cross alerting the subjects to be ready to detect the presence of a target, their attention would be already allocated equally to both hemifields, and thus, the presentation of the same stimulus on both sides, regardless of its affective value, would have no further effect on the distribution of attentional resources. This could also account for the lack of significant activation of the attention network in the comparison between $\mathrm{CS}+$ and $\mathrm{CS}-$. Another possibility is that a putative reduction in RTs to the $\mathrm{CS}+$, due to enhanced attention, was counterbalanced by an interference effect. It has been shown that the presentation 
of stimuli with aversive value can interfere with an ongoing unrelated task, resulting in longer response times to the task (e.g. [60]). The experimental paradigm used in the present study does not allow us to argue for either of these, or other, possible interpretations.

Stormark and co-workers [55,56], employing a similar procedure as the one used here, reported a reduced cost of processing targets invalidly cued by a $\mathrm{CS}+$. This result appears to contradict our findings. However, a major difference between the two studies was the duration of the CS+: in the Stormark and co-workers study the $\mathrm{CS}+$ was shown for $600 \mathrm{~ms}$, whereas in the present study the duration was only $50 \mathrm{~ms}$. Previous studies have shown that when the cue is present for longer than $300-500 \mathrm{~ms}$, reaction times to stimuli in the same location are actually slower, a phenomenon known as inhibition of return (IOR) [47]. It is, thus, possible that the results obtained by Stormark et al. reflect an IOR elicited by the conditioned stimulus. However, other reasons for the discrepancy between the two studies are possible and more studies (for example, varying in a parametric fashion the duration of the $\mathrm{CS}+$ ) would be necessary to further elucidate this issue.

The modulation of attention by the CS+ observed in the behavioural responses was associated with the activation of a distributed neural network that largely overlaps with the fronto-parietal network consistently proposed as having a key role in spatial attention and reported in previous neuroimaging studies (e.g. $[13,14,16,27,29,46,52]$ ), as well as bilateral activation of lateral OFC (see following sections). This pattern of activations provides direct support to the hypothesis that attention can be captured by stimuli that signal threat, such as the CS+ in this study, in an automatic fashion. It is important to note that in all trials, regardless of the attention condition, subjects were performing the same target detection task, for which the face stimuli were irrelevant. The key difference between experimental conditions was that in the case of neutral or divided attention condition (same stimulus in both hemifields), there was no further modulation of spatial attention by the face stimuli, whereas in the congruent and incongruent trials, subjects' spatial attention was shifted towards the location of the CS+ as demonstrated by the reaction times described above. Thus, the activations reported in Table 2 correspond to neural activity above and beyond that associated with target detection and motor responses.

The activation of the anterior aspect of the IPS (see Table 2) extended into the post-central sulcus. The spatial resolution of our images does not allow us to assess whether this activation lies entirely within the parietal lobe or in fact it also reflects some somatosensory activity. This latter possibility is particularly interesting given the suggested role of somatosensory cortex in emotional evaluation (e.g. [17]), and should be the focus of future investigation.

The orbitofrontal activation observed when attention was influenced by an emotional stimulus is intriguing, as activation of this region of prefrontal cortex has not been previously reported in neuroimaging studies of attention (for review see [7]) or fear conditioning [5,6,44]. Lateral OFC is ideally placed to provide an interface between these two processes, as projections from the amygdala to the lateral OFC are well documented in several species $[8,32,36,40]$, and important connections exist between the OFC, particularly the anterior lateral region, and the posterior parietal cortex and frontal eye fields [9,40,41]. Therefore, cells in the OFC can relay information about the affective value of a given stimulus in the environment from the amygdala to cortical areas subserving attention. Future studies may help to further elucidate the role of lateral $\mathrm{OFC}$ in the modulation of attention by emotional stimuli.

\subsection{Congruent versus incongruent trials}

The event-related nature of our experiment allowed us to separate the attention trials between congruent and incongruent. Such analysis revealed a significant activation in orbitofrontal cortex during incongruent trials, consistent with previous studies $[15,45]$. As mentioned above, this activation occurred in an area of OFC different, more ventral and posterior, from that activated in the focused-attention conditions, putatively in the posterior orbital gyrus (see [10]). Thus, it appears that different regions within lOFC are involved in the capture of attention by emotional stimuli and the "breaches of expectation" [45] that may subsequently occur.

In summary, we have shown that stimuli which acquired aversive value through fear conditioning can capture subjects' attention to their location. Furthermore, our study demonstrates that this modulation of attention by conditioned stimuli engages the fronto-parietal neural network thought to underlie the control of spatial attention, as well as the anterior $1 \mathrm{OFC}$.

\section{Acknowledgements}

We thank K. Mogg and B. Bradley for valuable discussions, D. McGonigle and C. Büchel for help with data analysis, and P. Vuilleumier for helpful comments on an earlier version of this manuscript. This work was funded by the Wellcome Trust. J.L.A. is supported by the Brain Research Trust.

\section{References}

[1] Armony JL, LeDoux JE. How danger is encoded: towards a systems, cellular, and computational understanding of cognitive-emotional interactions. In: Gazzaniga MS, editor. The new cognitive neurosciences. 2nd ed. Cambridge: MIT Press, 2000. p. 1067-79.

[2] Armony JL, Quirk GJ, LeDoux JE. Differential effects of amygdala lesions on early and late plastic components of auditory cortex spike trains during fear conditioning. Journal of Neuroscience 1998;18:2592-601. 
[3] Bradley BP, Mogg K, Millar N, Bonham-Carter C, Fergusson E, Jenkins J, et al. Attentional biases for emotional faces. Cognition and Emotion 1997;11:25-42.

[4] Büchel C, Dolan RJ. Classical fear conditioning in functional neuroimaging. Current Opinion in Neurobiology 2000;10:219-23.

[5] Büchel C, Dolan RJ, Armony JL, Friston KJ. Amygdala-hippocampal involvement in human aversive trace conditioning revealed through event-related functional magnetic resonance imaging. Journal of Neuroscience 1999;19:10869-76.

[6] Büchel C, Morris J, Dolan RJ, Friston KJ. Brain systems mediating aversive conditioning: an event-related fMRI study. Neuron 1998;20:947-57.

[7] Cabeza R, Nyberg L. Imaging cognition II: an empirical review of 275 PET and fMRI studies. Journal of Cognitive Neuroscience 2000;12:1-47.

[8] Carmichael ST, Price JL. Limbic connections of the orbital and medial prefrontal cortex in macaque monkeys. Journal of Comparative Neurology 1995;363:615-41.

[9] Cavada C, Goldman-Rakic PS. Posterior parietal cortex in rhesus monkey. II. Evidence for segregated corticocortical networks linking sensory and limbic areas with the frontal lobe. Journal of Comparative Neurology 1989;287:422-45.

[10] Chiavaras MM, LeGoualher G, Evans A, Petrides M. Three-dimensional probabilistic atlas of the human orbitofrontal sulci in standardised stereotaxic space. Neuroimage 2001;13:479-96.

[11] Chiavaras MM, Petrides M. Orbitofrontal sulci of the human and macaque monkey brain. Journal of Comparative Neurology 2000;422:35-54

[12] Convit A, McHugh P, Wolf OT, de Leon MJ, Bobinski M, De Santi $\mathrm{S}$, et al. MRI volume of the amygdala: a reliable method allowing separation from the hippocampal formation. Psychiatry Research 1999;90:113-23.

[13] Corbetta M, Kincade JM, Ollinger JM, McAvoy MP, Shulman GL. Voluntary orienting is dissociated from target detection in human posterior parietal cortex. Nature Neuroscience 2000;3:292-7.

[14] Corbetta M, Miezin FM, Shulman GL, Petersen SE. A PET study of visuospatial attention. Journal of Neuroscience 1993;13:1202-26.

[15] Coull JT, Frith CD, Buchel C, Nobre AC. Orienting attention in time: behavioural and neuroanatomical distinction between exogenous and endogenous shifts. Neuropsychologia 2000;38:808-19.

[16] Coull JT, Nobre AC. Where and when to pay attention: the neural systems for directing attention to spatial locations and to time intervals as revealed by both PET and fMRI. Journal of Neuroscience 1998;18:7426-35.

[17] Damasio AR, Grabowski TJ, Bechara A, Damasio H, Ponto LL, Parvizi J, et al. Subcortical and cortical brain activity during the feeling of self-generated emotions. Nature Neuroscience 2000;3:1049-56.

[18] Davis M. The mammalian startle response. In: Eaton RC, editor. Neural mechanisms of startle behaviour. New York: Plenum, 1984 p. 287-351.

[19] Davis M. The role of the amygdala in conditioned fear. In: Aggleton JP, editor. The amygdala: neurobiological aspects of emotion, memory, and mental dysfunction. New York: Wiley, 1992 p. 255-306.

[20] Ekman P, Friesen WV. Pictures of facial affect. Palo Alto (CA): Consulting Psychologists Press, 1976.

[21] Esteves F, Dimberg U, Öhman A. Automatically elicited fear: conditioned skin conductance responses to masked facial expressions. Cognition and Emotion 1994;8:393-413.

[22] Evans AC, Kamber M, Collins DL, Macdonald D. An MRI-based probabilistic atlas of neuroanatomy. In: Shorvon S, Fish D, Andermann F, Bydder GM, Stefan H, editors. Magnetic resonance scanning and epilepsy. New York: Plenum, 1994. p. 263-74.

[23] Fendt M, Fanselow MS. The neuroanatomical and neurochemical basis of conditioned fear. Neuroscience and Biobehaviour Review 1999;23:743-60.
[24] Filipek PA, Richelme C, Kennedy DN, Caviness Jr VS. The young adult human brain: an MRI-based morphometric analysis. Cerebral Cortex 1994;4:344-60.

[25] Friston KJ, Fletcher P, Josephs O, Holmes A, Rugg MD, Turner R. Event-related fMRI: characterising differential responses. Neuroimage 1998;7:30-40.

[26] Friston KJ, Holmes AP, Poline J-B, Frith CD, Frackowiak RSJ. Statistical parametric maps in functional imaging: a general linear approach. Human Brain Mapping 1995;2:189-210.

[27] Gitelman DR, Nobre AC, Parrish TB, LaBar KS, Kim YH, Meyer JR, et al. A large-scale distributed network for covert spatial attention: further anatomical delineation based on stringent behavioural and cognitive controls. Brain 1999;122:1093-106.

[28] Hartikainen KM, Ogawa KH, Knight RT. Transient interference of right hemispheric function due to automatic emotional processing. Neuropsychologia 2000;38:1576-80.

[29] Hopfinger JB, Buonocore MH, Mangun GR. The neural mechanisms of top-down attentional control. Nature Neuroscience 2000;3:284-91.

[30] Josephs O, Turner R, Friston K. Event-related fMRI. Human Brain Mapping 1997;5:243-8.

[31] Koch M. The neurobiology of startle. Progress in Neurobiology 1999;59:107-28.

[32] Krettek JE, Price JL. Projections from the amygdaloid complex to the cerebral cortex and thalamus in the rat and cat. Journal of Comparative Neurology 1977;172:687-722.

[33] LaBar KS, Gatenby JC, Gore JC, LeDoux JE, Phelps EA. Human amygdala activation during conditioned fear acquisition and extinction: a mixed-trial fMRI study. Neuron 1998;20:937-45.

[34] LeDoux, J. The emotional brain. New York: Simon \& Schuster, 1996

[35] LeDoux JE. Emotion circuits in the brain. Annual Review of Neuroscience 2000;23:155-84

[36] McDonald AJ. Organisation of amygdaloid projections to the prefrontal cortex and associated striatum in the rat. Neuroscience 1991;44:1-14

[37] Mesulam MM. Large-scale neurocognitive networks and distributed processing for attention, language, and memory. Annals of Neurology 1990;28:597-613.

[38] Mogg K, Bradley BP. Orienting of attention to threatening facial expressions presented under conditions of restricted awareness. Cognition and Emotion 1999;13:713-40.

[39] Mogg K, Mathews A, Eysenck M. Attentional bias to threat in clinical anxiety states. Cognition and Emotion 1992;6:149-59.

[40] Morecraft RJ, Geula C, Mesulam MM. Cytoarchitecture and neural afferents of orbitofrontal cortex in the brain of the monkey. Journal of Comparative Neurology 1992;323:341-58.

[41] Morecraft RJ, Geula C, Mesulam MM. Architecture of connectivity within a cingulo-fronto-parietal neurocognitive network for directed attention. Archives of Neurology 1993;50:279-84.

[42] Morris JS, Büchel C, Dolan RJ. Parallel neural responses in amygdala subregions and sensory cortex during implicit fear conditioning. Neuroimage 2001;13:1044-52.

[43] Morris JS, Friston KJ, Dolan RJ. Experience-dependent modulation of tonotopic neural responses in human auditory cortex. Proceedings of the Royal Society of London B: Biological Sciences 1998;265:649-57.

[44] Morris JS, Öhman A, Dolan RJ. Conscious and unconscious emotional learning in the human amygdala. Nature 1998;393:467-70.

[45] Nobre AC, Coull JT, Frith CD, Mesulam MM. Orbitofrontal cortex is activated during breaches of expectation in tasks of visual attention. Nature Neuroscience 1999;2:11-2.

[46] Nobre AC, Sebestyen GN, Gitelman DR, Mesulam MM, Frackowiak RS, Frith CD. Functional localisation of the system for visuospatial attention using positron emission tomography. Brain 1997;120: $515-33$.

[47] Posner MI. Orienting of attention. Quarterly Journal of Experimental Psychology 1980;32:3-25. 
[48] Pruessner JC, Li LM, Serles W, Pruessner M, Collins DL, Kabani N, et al. Volumetry of hippocampus and amygdala with high-resolution MRI and three-dimensional analysis software: minimising the discrepancies between laboratories. Cerebral Cortex 2000;10:433-42.

[49] Quirk GJ, Armony JL, LeDoux JE. Fear conditioning enhances different temporal components of tone-evoked spike trains in auditory cortex and lateral amygdala. Neuron 1997;19:613-24.

[50] Quirk GJ, Repa C, LeDoux JE. Fear conditioning enhances short-latency auditory responses of lateral amygdala neurons: parallel recordings in the freely behaving rat. Neuron 1995;15:1029-39.

[51] Repa JC, Muller J, Apergis J, Desrochers TM, Zhou Y, LeDoux JE. Two different lateral amygdala cell populations contribute to the initiation and storage of memory. Nature Neuroscience 2001;4: $724-31$.

[52] Rosen AC, Rao SM, Caffarra P, Scaglioni A, Bobholz JA, Woodley SJ, et al. Neural basis of endogenous and exogenous spatial orienting: a functional MRI study. Journal of Cognitive Neuroscience 1999;11:135-52.

[53] Rosen JB, Hitchcock JM, Sananes CB, Miserendino MJ, Davis M. A direct projection from the central nucleus of the amygdala to the acoustic startle pathway: anterograde and retrograde tracing studies. Behavioural Neuroscience 1991;105:817-25.
[54] Roskos-Ewoldsen DR, Fazio RH. On the orienting value of attitudes: Attitude accessibility as a determinant of an object's attraction of visual attention. Journal of Personality and Social Psychology 1992;63:198-211.

[55] Stormark KM, Hugdahl K. Conditioned emotional cueing of spatial attentional shifts in a go/do not go RT task. Intrenational Journal of Psychophysiology 1997;27:241-8.

[56] Stormark KM, Hugdahl K, Posner MI. Emotional modulation of attention orienting: a classical conditioning study. Scandinavian Journal of Psychology 1999;40:91-9.

[57] Talairach P, Tournoux J. Stereotactic coplanar atlas of the human brain. Stuttgart (Germany): Thieme, 1988.

[58] Vuilleumier P, Armony JL, Driver J, Dolan RJ. Effects of attention and emotion on face processing in the human brain: an event-related fMRI study. Neuron 2001;30:829-41.

[59] Weinberger NM. Physiological memory in primary auditory cortex: characteristics and mechanisms. Neurobiology of Learning and Memory 1998;70:226-51.

[60] Williams JM, Mathews A, MacLeod C. The emotional stroop task and psychopathology. Psychology Bulletin 1996;120:3-24.

[61] Worsley KJ, Friston KJ. Analysis of fMRI time series revisited again. Neuroimage 1995;2:173-81. 